\title{
Evaluación del impacto de una política pública: Ampliación del acceso a terapia antiretroviral en Chile
}

\author{
MINISTERIO DE SALUD, COMISIÓN NACIONAL DEL SIDA
}

\section{Impact evaluation of a public policy: Expanding access to antiretroviral therapy}

The Comisión Nacional de SIDA-CONASIDA (Chilean AIDS National Comission) directs its efforts into prevention campaigns and medical assistance to HIV positive persons. The general policy for their assistance has been to optimize resources, to make a national protocol of antiretroviral therapy (ARVT) with the advice of scientific societies, to unify the acquisition of antiretroviral drugs and to deal with the pharmaceutical companies their prices. About 80 percent of patients are assisted by the national public health system and the resting 20 percent concur to private medicine services. Anti retroviral therapy has been optimized as follows: in 1992 mono-therapy was started, in 1997 it was changed to bi-therapy and in 1999 tri-therapy begun. In $20025080 \mathrm{HIV}$ positive persons were under control by the public health system and in december 2000 tri-therapy reached $91,2 \%$ of coverage in whom it was prescribed. The positive impact of this program has been reflected in a 39 percent reduction of hospitalization, the increasing access to CD4 and viral load periodical determinations, delay in AIDS appearance and diminishing deaths due to AIDS.

Key words: AIDS; National program; Antiretroviral therapy; Impact

\section{Introducción}

La Comisión Nacional del SIDA es el organismo técnico del gobierno responsable de promover y facilitar los procesos destinados a que la sociedad asuma la prevención y disminución del impacto biosicosocial del VIH/SIDA.

Encargada de dictar las políticas públicas que se desarrollan en el tema, ha privilegiado dos ejes inseparables: prevención y atención, incluyendo en esta última el acceso a tratamiento antiretroviral (TARV).

Para la puesta en marcha de esta política se han empleado diversas estrategias que han permitido mejorar progresivamente la calidad y la cantidad de las terapias disponibles en el sector público de salud. Entre ellas se pueden mencionar: aumento de recursos financieros desde el presupuesto nacional; aumento en la eficiencia en el uso de esos fondos, incluyendo compras centralizadas; negociación con compañías farmacéuticas, apoyada por ONUSIDA; definición con comité científico asesor, de un protocolo único nacional.

CONASIDA ha querido evaluar los impactos que ha logrado la política de acceso a TARV, dada la gran inversión de recursos, tanto financieros como por parte de los equipos de salud involucrados. En esta tarea se han interesado diferentes equipos de investigadores nacionales y extranjeros, quienes han acompañado el proceso de expansión del acceso a tratamiento en Chile, considerando que puede entregar aprendizajes replicables a otros países de ingreso medio.

Los impactos se pueden medir en dos períodos temporales, a mediano y largo plazo, comprendiendo en el mediano plazo los efectos clínicos de la TARV, incluidos los inmunológicos (recuentos de linfocitos T CD4), virológicos (carga viral - CV), aparición de enfermedades oportunistas, hospitalizaciones y retraso en aparición de la etapa SIDA. Otros impactos tienen que ver con efectos socioeconómicos tales como ahorro en el Sistema Nacional de Servicios de Salud.

El impacto más tardío o a largo plazo considera la mortalidad, la cual está fuertemente influenciada por la cobertura de acceso a TARV. Algunos estudios ya muestran cambios en la sobrevida y mortalidad de las personas viviendo con el VIH (PVVIH), que es necesario continuar evaluando, dado que al 31 de diciembre de 2002 se alcanzó cobertura de acceso a TARV de 91,2\%. 
Aumento del acceso a terapia antiretroviral, años 1993 a 2002. CONASIDA

La TARV moderna ha modificado la historia natural de la infección por VIH. Como se describió en la Conferencia Internacional de SIDA de Vancouver, en 1996, el mayor impacto se produce con triterapia. Se reducen complicaciones y hospitalizaciones, junto con disminuir las defunciones y mejorar globalmente la calidad de vida de las personas con TARV. Sin embargo, el alto costo de estas terapias ha determinado que su aplicación esté limitada a los países de altos recursos o a los grupos sociales que pueden solventar directamente este gasto.

Chile cuenta con un sistema de salud mixto, y de acuerdo con la distribución entre sectores público y privado, se estima que de las PVVIH en control de salud, $80 \%$ lo hace en el sector público y el $20 \%$ restante en el sector privado. Las PVVIH que requirieron tratamiento con TARV durante el año 2002 corresponden al 70,9\% del total de los casos controlados, de acuerdo con la información de los Servicios de Salud, cifra que tiende a descender por ingreso progresivo de personas en etapas precoces de la infección.

A lo largo del tiempo se ha incrementado el presupuesto destinado a medicamentos antiretrovirales, pero la mayor complejidad de esquemas y la incorporación de fármacos de precios más elevados significó durante algunos años una reducción de las coberturas, que se está revirtiendo a partir del año 2002.

Respecto a las coberturas, la cifra exacta de personas en control en el sistema público de salud y con indicación de tratamiento se conoce desde 2001. Previamente, a partir de 1993, se entregó monoterapia (AZT) al total de las PVVIH para quienes se solicitaba tratamiento a CONASIDA; ello se mantuvo hasta 1997, cuando el Ministerio de Salud empezó a proporcionar biterapia a la población beneficiaria del sistema público, con una reducción en las coberturas estimadas. Dos años más tarde, se adoptó la triterapia. El año 2001, la cobertura llegó a 56\% de las personas que solicitaron tratamiento. En esa cobertura, aproximadamente la mitad se mantuvo con biterapia y a la otra mitad se le asignó triterapia.

Estas biterapias en algunos casos eran complementadas con el tercer fármaco aportado por la familia, el entorno social de la persona, el municipio y otros. A partir del 2002 se entrega exclusivamente triterapia logrando una cobertura que alcanza a fines de diciembre al 91,2\% (Figura 1).

$\mathrm{El}$ aumento del acceso a las terapias provocó un ingreso progresivo de personas a control en el sistema público de Salud, que varió de 4.421 personas, el 2001, a 5.080, el 2002, cifra ligeramente inferior a las estimaciones previas, lo que permitió ajustar las proyecciones de demanda de terapia y descartar la hipótesis de una gran demanda oculta, no evidenciada por la falta de acceso a tratamiento.

Impacto clínico de la terapia antiretroviral Efectos inmunológicos. Evolución de los recuentos de linfocitos T CD4 en una cohorte, 1989 a 2001.

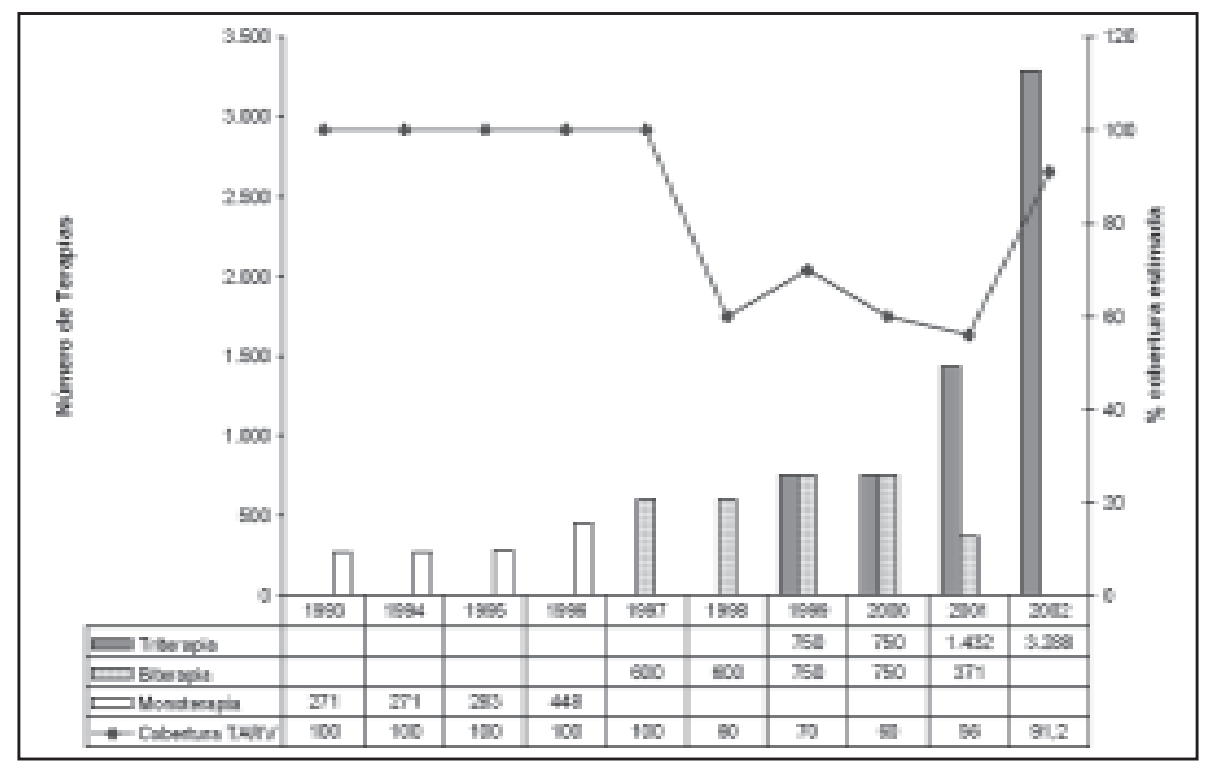

Figura 1. Número de PVVIH en TARV por tipo de terapia y cobertura de acceso. Chile, 19932002. CONASIDA/MINSAL. PVVIH: persona viviendo con el VIH. TARV: terapia antiretroviral. 
Evaluación financiada y realizada técnicamente por ANRS (Agence Nationale de Recherches sur le SIDA, de Francia) para la iniciativa de acceso a TARV de ONUSIDA/CONASIDA. (Información preliminar).

Los investigadores de la ANRS realizaron un estudio de cohorte en el Hospital Del Salvador

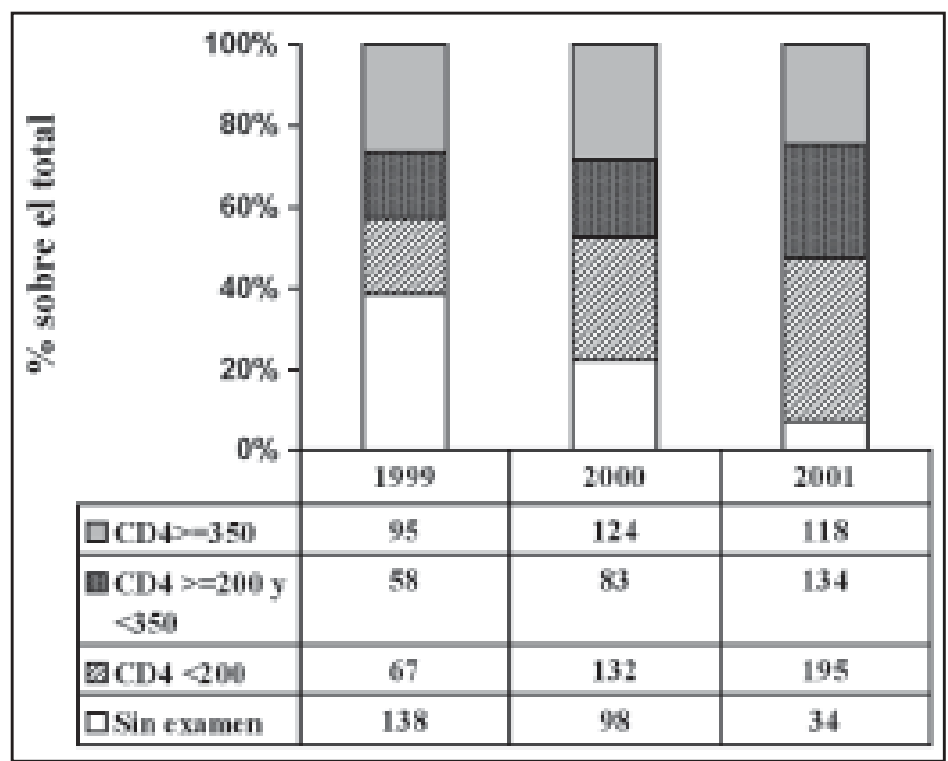

Figura 2. Última cifra conocida de recuento de linfocitos T CD4 entre las PVVIH en control activo, al 31 de diciembre de 1999, 2000 y 2001. Hospital Del Salvador, Región Metropolitana, Chile. ANRS, Evaluación ONUSIDA/ CONASIDA. Información preliminar. 2002.

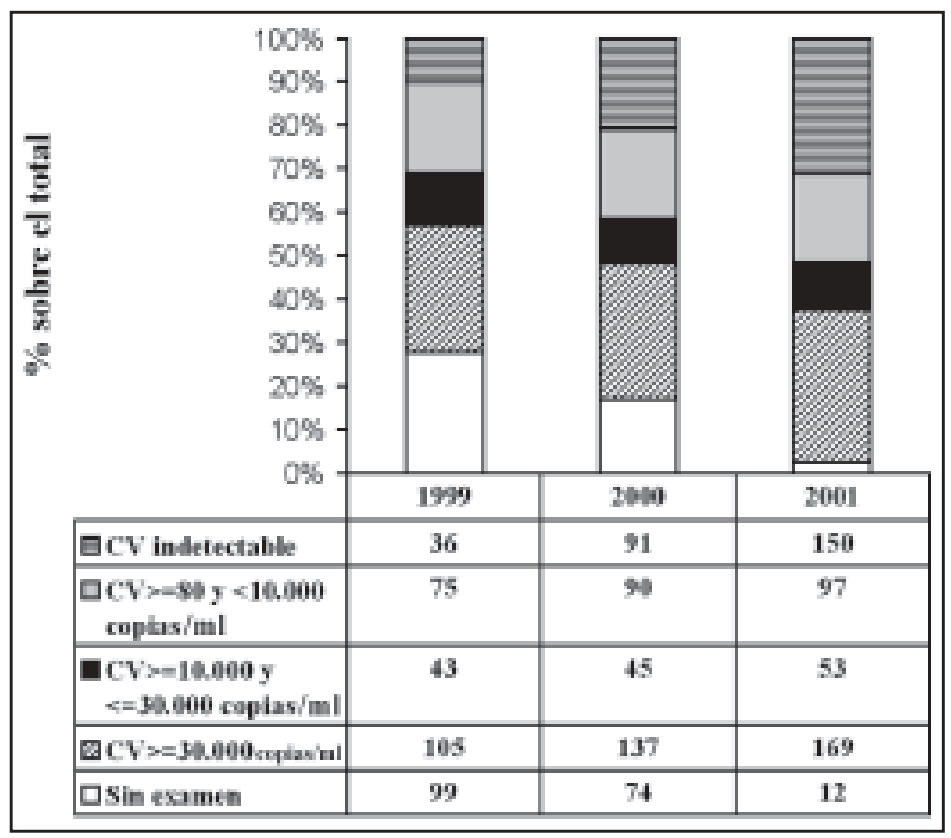

Figura 3. Carga viral (número de copias) PVVIH en control activo al 31 de diciembre de 1999, 2000 y 2001. Hospital Del Salvador, Región Metropolitana, Chile. ANRS, Evaluación ONUSIDA/CONASIDA. Información preliminar. 2002.
(S.S.M. Oriente), incorporando a PVVIH controladas entre el $1^{\circ}$ de enero de 1989 y el 31 de diciembre de 2001. De la información recolectada se analiza el acceso a recuentos de linfocitos $\mathrm{T}$ CD4 (Figura 2).

El mejor indicador para decidir el inicio a TARV en PVVIH asintomáticas es el recuento de CD4 (el Protocolo Nacional definía como uno de los criterios de ingreso a triterapia tener recuentos iguales o menores a $350 \mathrm{CD} 4 / \mathrm{mm}^{3}$ y, desde octubre de 2001, que sean inferiores a $200 \mathrm{CD} 4 / \mathrm{mm}^{3}$ ).

Los recuentos de CD4 para las PVVIH en control activo (igual o mayor a 6 meses) muestran la disminución de la categoría sin resultado desde $38,6 \%$ en 1999 a $7,1 \%$ el 2001, reflejando la ampliación en el acceso a la técnica. La alta proporción sin examen en los primeros años no permite hacer comparación de los resultados en el tiempo.

Efectos virológicos. Evolución de los resultados de la carga viral en una cohorte, 1989 a 2001.

Evaluación financiada y realizada técnicamente por ANRS (Francia) para la iniciativa de acceso a TARV de ONUSIDA/CONASIDA.

La carga viral es uno de los predictores de evolución, sin ser el principal, pero es imprescindible para monitorear la respuesta al tratamiento.

Se observa reducción de personas sin acceso a la técnica. La alta proporción sin examen en los primeros años no permite hacer comparación de los resultados en el tiempo (Figura 3).

\section{Reducción de hospitalizaciones, Estudio de cuentas nacionales, 1999 a 2000.}

Financiado y apoyado técnicamente por ONUSIDA - Funsalud/ Sidalac, (México) - CONASIDA (Chile).

Entre la información recolectada para este estudio se analizan todas las hospitalizaciones asociadas al VIH/SIDA producidas entre los años 1999 y 2000 en la Funda- 
ción San Borja-Arriarán, centro que concentra la mayor proporción de las PVVIH controladas en el país y en la Región Metropolitana.

Las hospitalizaciones por VIH/SIDA en este establecimiento presentaron una disminución general de $39 \%$ entre 1999 y 2000 . En la Tabla 1 se muestra las variaciones de las hospitalizaciones por diferentes patologías, siendo criptococosis, neumonía bacteriana y criptosporidiasis los diagnósticos que experimentaron mayor reducción.

La reducción de las hospitalizaciones constituye un indicador indirecto de la mejoría en la calidad de vida de las PVVIH.

Tabla 1. Hospitalizaciones de PVVIH por tipo de patología. Fundación San Borja Arriarán. 1999- 2000

\begin{tabular}{lrrr}
\hline Diagnóstico & $\mathbf{1 9 9 9}$ & $\mathbf{2 0 0 0}$ & Variación \% \\
\hline Acidosis láctica & 3 & 3 & 0 \\
Pneumocystis jiroveci (ex carinii) & 26 & 30 & +15 \\
Criptosporidiasis & 10 & 3 & -70 \\
Toxoplasmosis cerebral & 7 & 7 & 0 \\
Mycobacterium avium & 3 & 3 & 0 \\
Criptococosis & 26 & 3 & -88 \\
Citomegalovirus digestivo & 3 & 0 & \\
Tbc extrapulmonar & 13 & 7 & -46 \\
Linfoma no Hodgkin & 0 & 6 & \\
Candidiasis & 138 & 79 & -43 \\
Sarcoma de Kaposi & 26 & 13 & -50 \\
Neumonía bacteriana & 23 & 7 & -70 \\
Tbc pulmonar & 23 & 23 & 0 \\
Total & 301 & 184 & -39 \\
\hline Cuentas Nacionales. ONUSIDA, FUNSAL
\end{tabular}

Cuentas Nacionales. ONUSIDA, FUNSALUD, CONASIDA/MINSAL.

\section{Retraso en la aparición de la etapa SIDA, 1984 a 2001. CONASIDA.}

El análisis epidemiológico muestra una tendencia a la disminución en la aparición de SIDA, que se inició a partir del año 1998 con una reducción respecto al año anterior, producto de la incorporación de la biterapia. Al observar la incidencia de SIDA se aprecia que esta disminución de la tasa es sostenida y, en el año 2000, se produjo una disminución aún mayor, probablemente producto de la incorporación de la triterapia. También se observó, a partir de 1997, una separación de las curvas de incidencia de SIDA y VIH, alcanzando un máximo el año 2000 (Figura 4).

Al analizar el ritmo de crecimiento de la infección asintomática, en períodos quinquenales y en términos porcentuales, vemos que el quinquenio 1996-2001 presentó una reducción de $7,2 \%$ con respecto al período anterior; en tanto, el quinquenio 1990-1995 disminuyó en 23,9\% con relación al período $1984-$ 1989. Por lo tanto, existe una tendencia en el tiempo a la reducción del ritmo de crecimiento de la incidencia del VIH, fenómeno que se intensificó en el quinquenio 19962001.

Un análisis similar sobre el ritmo de crecimiento del SIDA nos muestra que, durante el quinquenio 1996-

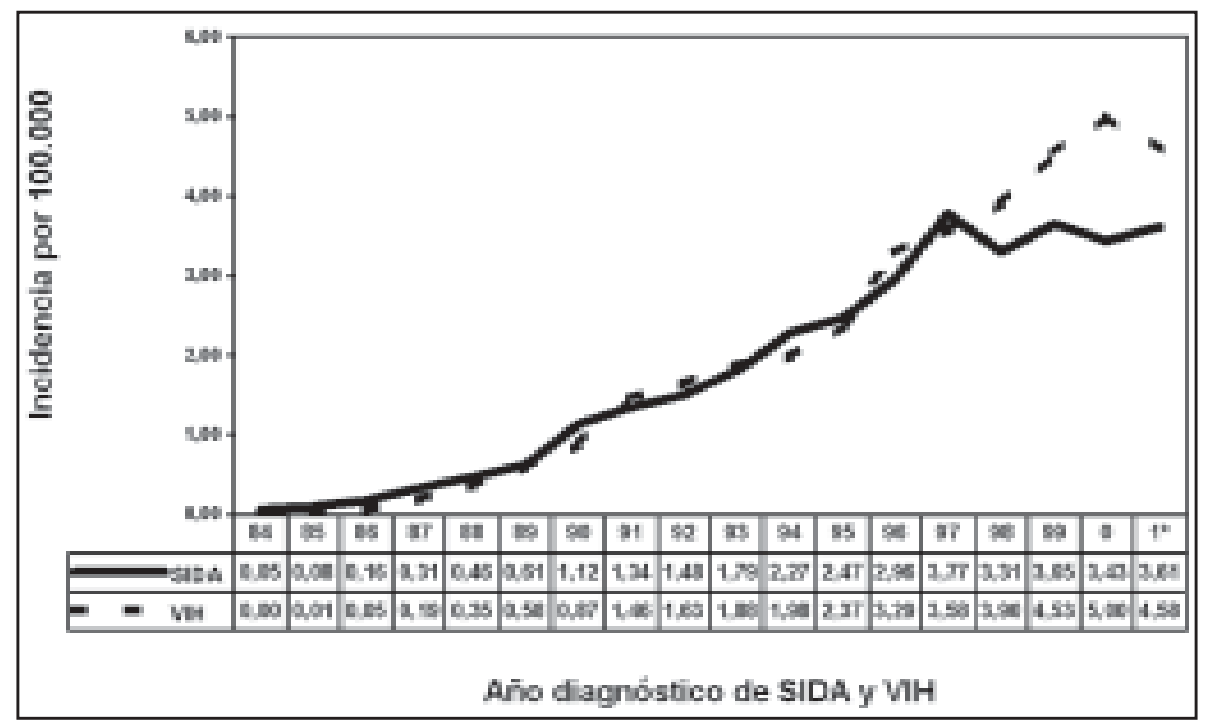

Figura 4. Incidencia de SIDA e infección por VIH por año de diagnóstico. Chile. 1984-2001. Información al 31 diciembre 2001. Proyecciones de población INE, Censo 1992. (*) Cifra preliminar debido a latencia en la notificación. CONASIDA-MINSAL. 
2001 se produjo una disminución promedio de $4,9 \%$ con relación al período $1990-1995$, quinquenio que a su vez presentó un descenso de $17,3 \%$ respecto al de 1984-1989. Entonces, también se observa una tendencia a la disminución del ritmo de incremento de la incidencia de SIDA, proceso que se intensificó en el quinquenio 19962001.

\section{Las defunciones por SIDA}

Mortalidad por SIDA en la población general, 1984 a 2001. Departamento de Estadística MINSAL - CONASIDA.

Se analiza la tendencia de la mortalidad por SIDA en la población general. La curva muestra una tendencia al aumento, llegando a una tasa de 2,9 por 100.000 habitantes, en 1997. A partir de 1998 se observó una disminución mantenida hasta el año 2000 (Figura 5).

En la figura se asocia la curva de mortalidad anual corregida por subnotificación, mostrando la diferencia entre ésta y la mortalidad observada, así como el impacto atribuible a la biterapia (transitorio) y a la triterapia parcial. Se excluye el año 1999, para el cual no fue posible hacer este proceso de corrección.

No es posible evidenciar todavía el efecto de la expansión del acceso a triterapia del 2002 en la mortalidad.

\section{Letalidad en las personas viviendo con SIDA, 1984 a 2001. CONASIDA}

La letalidad, referida al porcentaje de casos fallecidos del total de personas con SIDA vivas durante el año, fue elevada durante los primeros años de epidemia, estabilizándose desde fines de los 80 en cifras cercanas al $30 \%$ anual. A partir de 1998 se observó un descenso en la letalidad que puede atribuirse al efecto del acceso a TARV (Figura 6).

Aunque para el 2001 la cifra es preliminar, se observó un incremento de la letalidad apoyando la transitoriedad del efecto de la biterapia y el impacto que tiene en ambos indicadores la cobertura de la terapia, esperándose que el incremento de TARV del 2002 se apreciará en el futuro.

\section{Años de vida potenciales perdidos por SIDA, 1984 a 2001. CONASIDA}

Debido a que las personas afectadas por el SIDA son en su mayoría jóvenes, su muerte impacta fuertemente en el indicador Años de Vida Potenciales Perdidos (AVPP), que se refiere a los años que la persona perdió de vivir debido a muerte prematura. La tendencia muestra un incremento hasta 1997, descendiendo un $42,0 \%$ entre los años 1998 y 2000. Esta marcada reducción se puede atribuir a la incorporación de mejores alternativas de tratamiento (biterapia). También se observa transitoriedad de este efecto, subiendo el indicador para el año 2001, aún cuando la información para ese año es incompleta (Figura 7).

El impacto del aumento del acceso a triterapia en este parámetro se debería evidenciar en los próximos años.

\section{Primeras causas de muerte. Año 2000. \\ Departamento Estadística Minsal-CONASIDA}

El siguiente análisis se refiere a las primeras causas de muerte en Chile, en hombres y mujeres de 20 a 44 años. La fuente utilizada es el

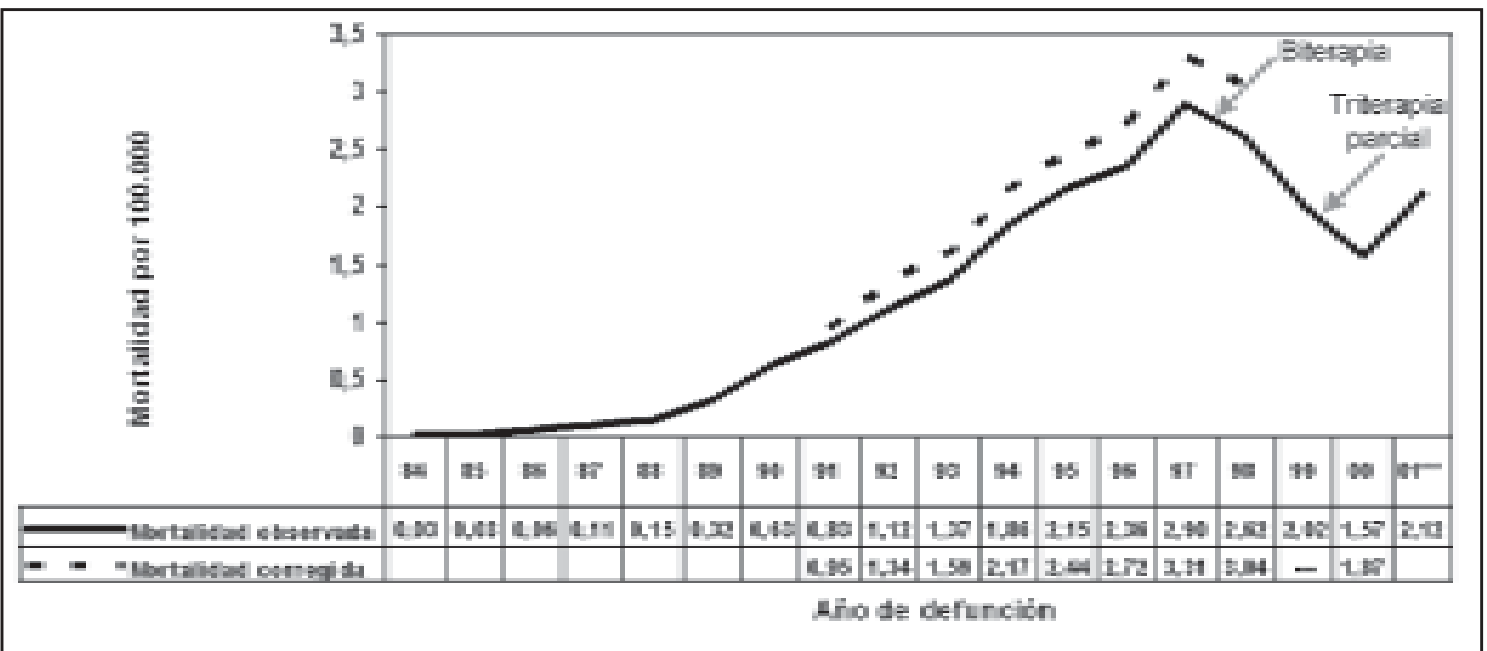

Figura 5. Mortalidad por SIDA corregida* Chile. 1984-2001. Información al 31 de diciembre 2001. Proyecciones de población INE, Censo 1992. (*) Mortalidad corregida por subnotificación. (2001**) Cifra preliminar debido a latencia en la información. Estadística - CONASIDA. MINSAL. 


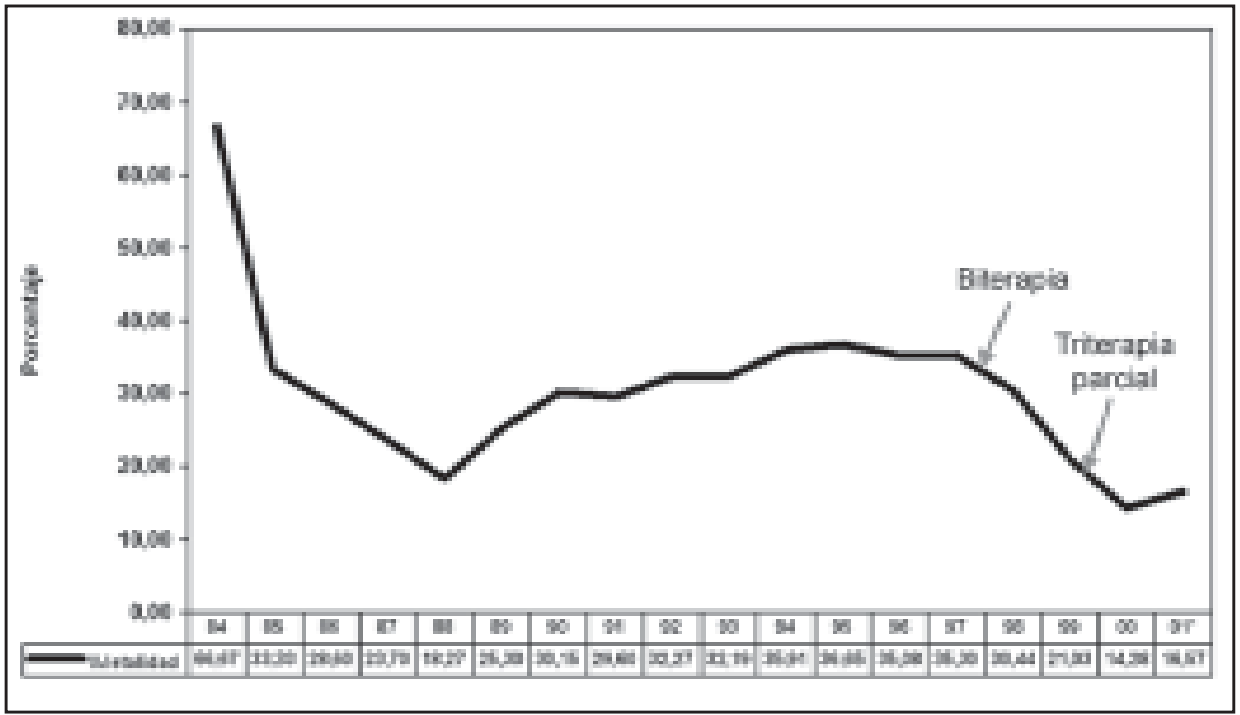

Figura 6. Letalidad por SIDA. Chile. 1984-2001. Información al 31 de diciembre 2001. (*) Cifra preliminar debido a latencia de la información. CONASIDA. MINSAL.

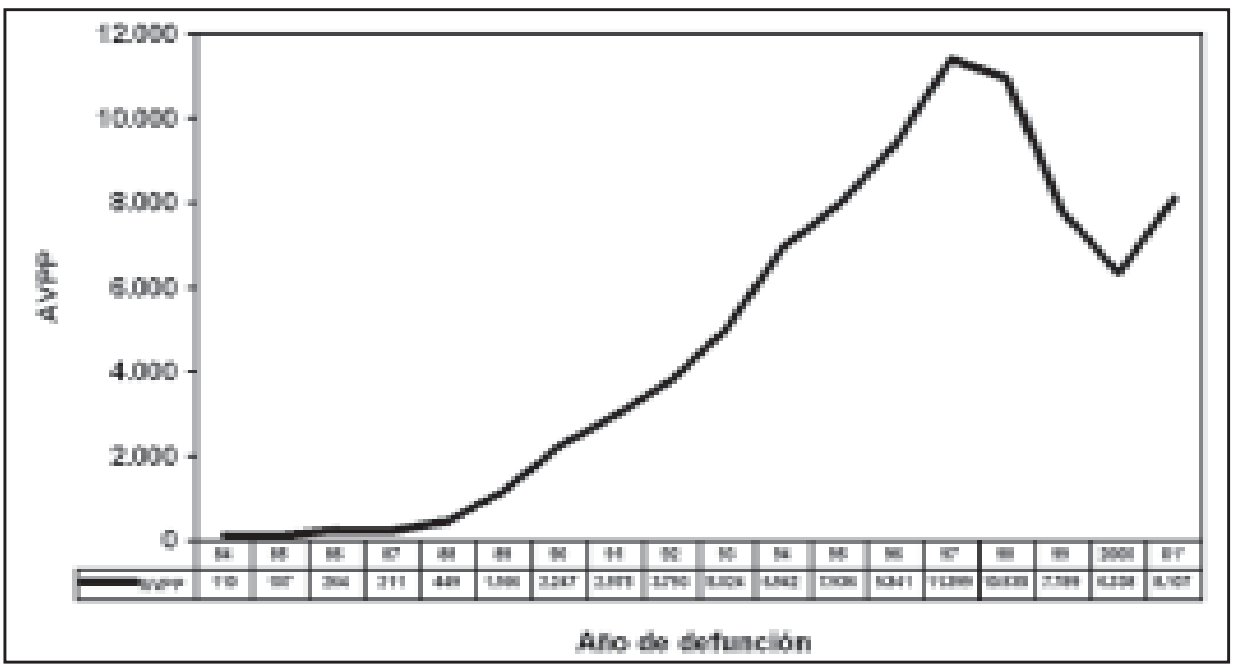

Figura 7. Años de vida potenciales perdidos (AVPP) por SIDA en hombres de 20 a 49 años. Chile. 1984-2001. Información al 31 de diciembre 2001. (*) Cifra preliminar debido a latencia en la notificación. CONASIDA. MINSAL.

registro nacional de mortalidad, basado en la Clasificación Internacional de Enfermedades (CIE 10), implementado por convenio tripartito entre el Servicio de Registro Civil e Identificación, el Instituto Nacional de Estadísticas y realizada por el Departamento de Estadística del Ministerio de Salud. Las causas de muerte comprendidas entre los códigos B20 y B24 corresponden a SIDA.

Durante el 2000, el SIDA ocupó el cuarto lugar en el ámbito nacional como causa específica de muerte entre hombres de 20 a 44 años, con una tasa de 10,0 por 100.000 hombres de esa edad. Las causas que le antecedieron corresponden principalmente a muertes por accidentes de transporte, lesiones infligidas intencionalmente y agresiones. Entre las diez primeras causas de defunción es la única de tipo transmisible (Tabla 2).

En la Región Metropolitana, la mortalidad por 10 primeras causas en hombres de igual grupo de edades muestra que el SIDA se ubicó en cuarto lugar, con una tasa de 14,9 por 100.000 hombres de 20 a 44 años y fue precedido por accidentes 
Tabla 2. Principales causas de muerte en hombres de 20 a 44 Años. Chile, 2000

\begin{tabular}{lccr}
\hline Causa de defunción (CIE-10) & Defunciones & Tasa* & \% \\
\hline Accidentes de transporte & 864 & 29,3 & 16,1 \\
Lesiones infligidas intencionalmente & 689 & 23,3 & 12,8 \\
Agresiones & 446 & 15,1 & 8,3 \\
& & & \\
SIDA & 296 & 10,0 & 5,5 \\
Causas externas de intención no determinada & 259 & 8,8 & 4,8 \\
Enfermedades del hígado & 257 & 8,7 & 4,8 \\
Exposición a factores no especificados & 173 & 5,9 & 3,2 \\
Ahogamiento y sumersión accidentales & 162 & 5,5 & 3,0 \\
Enfermedades isquémicas del corazón & 138 & 4,7 & 2,6 \\
Enfermedades cerebrovasculares & 131 & 4,4 & 2,4 \\
Acumulado primeras 10 causas & 3.415 & 115,7 & 63,6 \\
Resto de causas & 1.956 & 66,2 & 36,4 \\
Total de causas & 5.371 & 181,9 & 100,0 \\
\hline
\end{tabular}

Fuente: Ministerio de Salud, Departamento de Estadísticas e Información de Salud. Tasa por 100.000 hombres de 20 a 44 años. Proyecciones de población INE, Censo 1992.

Tabla 3. Principales causas de muerte en hombres de 20 a 44 Años. Región Metropolitana, año 2000

\begin{tabular}{lrrr}
\hline Causa de defunción (CIE-10) & Defunciones & Tasa* & \% \\
\hline Accidentes de transporte & 301 & 25,6 & 16,0 \\
Lesiones infligidas intencionalmente & 236 & 20,0 & 12,5 \\
Agresiones & 207 & 17,6 & 11,0 \\
SIDA & 175 & 14,9 & 9,3 \\
Enfermedades del hígado determinada & 90 & 7,7 & 4,8 \\
Otras enfermedades del corazón & 64 & 5,5 & 3,4 \\
Envenenamiento accidental por y exposición & & & \\
a sustancias nocivas & 45 & 3,8 & 2,4 \\
Otras causas externas de traumatismos & & & \\
accidentales & 31 & 2,6 & 1,6 \\
Ahogamiento y sumersión accidentales & 30 & 2,6 & 1,6 \\
Enfermedades isquémicas del corazón & 29 & 2,5 & 1,5 \\
Acumulado primeras 10 causas & 1.208 & 102,9 & 62,5 \\
Resto de causas & 674 & 57,4 & 37,5 \\
Total de causas & 1.882 & 160,3 & 100,0 \\
\hline
\end{tabular}

Fuente: Ministerio de Salud, Departamento de Estadísticas e Información de Salud. Tasa por 100.000 hombres de 20 a 44 años. Proyecciones de población INE, Censo 1992.

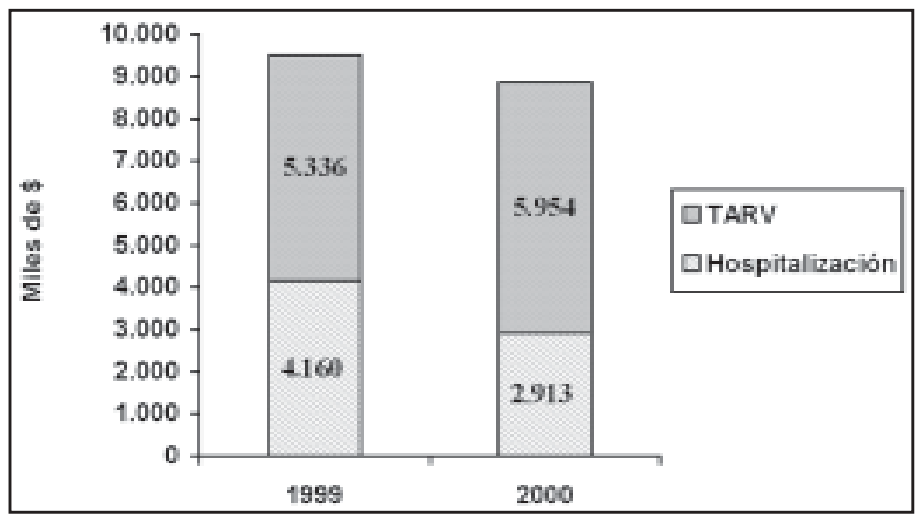

Figura 8. Recursos financieros destinados a hospitalización y TARV para PVVIH. Chile, 1999-2000. Cuentas Nacionales. ONUSIDA, FUNSALUD, CONASIDA/MINSAL. Chile. 1999-2000. de transporte, lesiones infligidas intencionalmente y agresiones (Tabla 3 ).

En mujeres, el SIDA no figura entre las diez primeras causas de muerte del país ni de la Región Metropolitana. El número de afectadas es aún reducido en comparación con las que fallecen por las enfermedades que constituyen las diez primeras causas de muerte en mujeres de este grupo de edades: accidentes de transporte, tumores malignos de cuello del útero y de mama y enfermedades cerebro vasculares.

\section{Impacto financiero de la ampliación del acceso a TARV, 1999 a 2000}

Estudio de Cuentas Nacionales. Financiado y apoyado técnicamente por ONUSIDA - Funsalud/Sidalac, (México) - CONASIDA.

De acuerdo a este estudio, la inversión en TARV representa la mayor proporción del gasto total en VIH/SIDA, correspondiendo a $36 \%$ en 1999 y a $40 \%$, para el 2000. El aumento en el 2000 corresponde básicamente a la incorporación de la triterapia y al incremento de la cobertura de acceso a tratamiento de las PVVIH del sector público.

El gasto en hospitalización, segundo en importancia, está determinado fundamentalmente por el peso relativo del valor de día cama (85\% del costo total de una hospitalización) por tratarse de pacientes con estadías hospitalarias de larga duración. Las cifras demuestran que un mayor gasto en TARV se correlaciona con una significativa disminución del gasto en hospitalización por enfermedades oportunistas (Figura 8).

La disminución en hospitalizaciones significó un ahorro de 1.255 millones de pesos el año 2000, para los establecimientos del sistema público.

A pesar del mayor gasto en antiretrovirales, en general se produjo un ahorro neto para el sistema público de Salud de 629 millones de pesos el año 2000. 


\section{Conclusiones}

La ampliación del acceso a TARV produjo, en un plazo sorprendentemente breve, cambios en los parámetros clínicos, demostrándose la mejoría en el estado inmunológico, descenso de las mediciones virológicas, reducción de hospitalizaciones y del paso a la etapa SIDA.

Al contrastar el ahorro debido al descenso de las hospitalizaciones con el mayor gasto en antiretrovirales, se aprecia un ahorro neto, ya que se trata de hospitalizaciones prolongadas y de alto costo por requerir habitualmente de ingreso a salas de alta complejidad.

El impacto en la mortalidad se hará evidente en los próximos años, ya que en esta etapa se refleja el acceso a biterapia y el inicio de la triterapia.

Es evidente que la política de acceso a TARV resulta favorable, en términos de costo-efectividad, al tener efectos clínicos que conllevan mejoría en la calidad de vida de las PVVIH y epidemiológicos que constituyen un aporte a la Salud Pública.

\section{Resumen}

La Comisión Nacional de SIDA-CONASIDA centra sus esfuerzos en prevención y atención de las personas infectadas por VIH. En la atención de los infectados, la política general ha sido optimizar recursos, elaborar un protocolo nacional de tratamiento único con el concurso de las sociedades científicas, centralizar la adquisición de medicamentos y negociar con sus productores los costos. Aproximadamente $80 \%$ de los pacientes son atendidos en el sector público y 20 restante en forma privada. La optimización en la terapia antiretroviral ha sido la siguiente: en 1992 inicio de monoterapia, en 1997, de la biterapia y en 1999 de la triterapia. El año 2002 se controlaban en el sector público 5080 personas infectadas por VIH y en diciembre de ese año ya se alcanzó $91,2 \%$ de cobertura con triterapia para quienes la necesitaron. El impacto positivo de este programa se ha reflejado en la reducción en 39\% de las hospitalizaciones, el acceso creciente a las determinaciones periódicas de CD4 y carga viral, el retraso en la aparición de SIDA y una disminución de las muertes causadas por el SIDA.

Correspondencia a:

Anabella Arredondo Paz.

E-mail: arre@minsal.cl 